\title{
Renal Sarcoidosis Presenting as Acute Kidney Injury with Granulomatous Interstitial Nephritis
}

\author{
Nadia Bouchemla ${ }^{1 *}$, Meriem Chettati ${ }^{2}$, Poda Anselme ${ }^{3}$, Meryem labrassi ${ }^{4}$, Wafaa Fadili ${ }^{5}$ Inass Laouad $^{6}$
}

${ }^{1}$ Resident, ${ }^{2}$ Associate Professor, ${ }^{3,4}$ Resident, ${ }^{5}$ Professor, ${ }^{6}$ Professor Department of Nephrology, Mohamed VI Medical Center, Marrakesh, Morocco

*Corresponding author: Nadia Bouchemla

Sarcoidosis is a nonnecrotizing granulomatous disease of unknown origin that can affect lungs, eyes, kidneys and skin. Although kidney involvement is usually clinically silent, granulomatous interstitial nephritis - the hallmark of renal sarcoidosis - can lead to functional impairment and organ failure. We report a case of renal sarcoidosis revealed by acute kidney injury with biopsy finding of a granulomatous interstitial nephritis. Outcome was favorable after the institution of high dose corticosteroids.

Keywords: Sarcoidosis, interstitial nephritis, noncaseating granuloma.

Copyright @ 2020: This is an open-access article distributed under the terms of the Creative Commons Attribution license which permits unrestricted use, distribution, and reproduction in any medium for non-commercial use (NonCommercial, or CC-BY-NC) provided the original author and source are credited.

\section{INTRODUCTION}

Sarcoidosis is a systemic disease of middleaged patients, with a prevalence of about 4.7-64 in 100000 , and an incidence of 1.0-35.5 in 100000 per year [1].This inflammatory disorder is characterized by noncaseating epithelioid cell granulomas commonly involving the lung and reticuloendothelial system [2].

Kidney disease develops in up to a third of patients, and may present as acute kidney injury related to hypercalcemia, or glomerular filtration rate (GFR) decline and low level proteinuria due to granulomatous parenchymal injury. Reported patterns of kidney injury are interstitial nephritis or glomerular disease [3]. We report a case of renal sarcoidosis revealed by acute kidney injury.

\section{Case Report}

A 58-year-old morocan female presented with acute kidney injury. The patient's medical history included productive cough, dyspnea and headache a month before. However, She was a teacher and reported no smoking, alcohol dependance or illicit drug use.

Clinical examination found a bood pressure of 160/8. Urine studies showed dipstick proteinuria (1+), no hematuria. Neurologic examination was normal.She had a erythema nodosom .Finding of the examination the heart, lungs, and abdomen were unremarkable.
The initial laboratory tests were notable for the following values: serum creatinine ( $\mathrm{SCr}$ ), $415 \mu \mathrm{mol} / \mathrm{L}$; serum calcium, $131 \mathrm{mmol} / \mathrm{L}$, serum albumin, $33 \mathrm{~g} / \mathrm{L}$; and hemoglobin, $9.8 \mathrm{~g} / \mathrm{dL}$. Proteinuria was $0.7 \mathrm{~g} / \mathrm{day}$ with no hematuria. Liver function tests were normal. Antineutrophil cytoplasmic antibody serologic test results were negative. The work up was negative for hypocomplementemia, monoclonal gammapathy, and viral serology. .Angiotensin-convertiong enzyme level was elevated in serum 199 UI.

Ultrasound showed normal kidney size and echogenicity with no evidence of obstruction or nephrolithiasis.A chest radiopraph showed mild diffuse interstitial disease.

Computed tomography showed 2 nodules and calcifications in the lung. Pulmonary function testing was normal. Angiotensin-convertiong enzyme level was elevated in serum. ANCA serologic test results were negative. Magnetic resonance imaging of the brain was normal. A kidney biopsy was performed to determine the cause of the AKI.

Light microscopic evaluation of the kidney biopsy specimen was performed with hematoxylin and eosin. There were 2 nonglobally sclerosed glomeruli, several of which showed focal ischemic tuft retraction. Approximately $30 \%$ of the renal cortex showed interstitial fibrosis, tubular atrophy, and interstitial lymphocytic and granulomatous inflammation (Figure A). There were multiple interstitial noncaseating 
granulomas comprised of lymphocytes and monocyts (Figure B). Routine immunofluorescence was negative for all immunoglobulins.

The patient was strarted on treatment with methylprednisolone, $500 \mathrm{mg}$, intravenously daily for 3 days, followed by oral prednisone, $0.5 \mathrm{mg} / \mathrm{kg} /$ day $\mathrm{mg}$. Durant the next month, her SCr level decreased to 123 $\mu \mathrm{mol} / \mathrm{L}$. After six months, she continues on maintenance prednisone therapy at $10 \mathrm{mg}$ daily, with the improvement of kidney function (serum creatinine: $123 \mu \mathrm{mo} / \mathrm{L})$.

\section{DisCUSSION}

Sarcoidosis was recognised more than 120 years ago, and it remains a confusing disease with many grey areas (4). The cause of sarcoidosis is not known and diagnosis can be difficult and delayed from the diverse, nonspecific presentations [1].

The notion of environmental or transmissible agents having a causal role in sarcoidosis is lent support by temporal and space-time clusters. Exposure to musty odours, insecticides, or to metal-processing industries is disease risk factors [5, 2]. Sarcoidosis is usually sporadic, but is familial in $3 \cdot 6-9 \cdot 6 \%$ of cases [6].

The disease can occur in both men and women, with $70 \%$ of patients aged 25-45 years; however, in Europe and Japan, a second peak of incidence occurs in women older than 50 years of age $[7,8]$.

The exact cause of sarcoidosis is still not known. Many studies suggest that the genitic susceptibility and environmental factors contribute to disease development $[9,2,10]$.

We present a case of a patient who had an AKI with a Lofgren syndrome suggestive of sarcoidosis. The kidney biopsy findings of granulomatous inflammation Such clinical presentation and the exclusion of other possible causes of granulomatous disease established a clinicalpathologic diagnosis of renal sarcoidosis.

Granulomatous inflammation can be observed in various conditions such us infections, lymphoma, or granulomatous with polyangiitis [11].

Granulomas characteristically consist of epitheloid cells and multinucleated giant cells with CD4 sponse. Over time, fibroblasts and mast cells lay down collagen and proteoglycans, causing fibrosis. At a single center, the histopathologic findings of interstitial nephritis with non-necrotizing granulomas were attributed to drugs (44\%), sarcoidosis (29\%), or granulomatosis with polyangiitis $(5 \%)[3,12]$.

Corticosteroid therapy is the treatment of choice in sarcoid-associated GIN, often resulting in significant improvement in kidney function [13]. Steroid therapy cessation or withdrawal frequently is associated with relapse of kidney injury that is reversed on reinstitution of the therapy [14]. Long-term lowdose steroid treatment (5-7.5 mg/d of prednisolone) may preserve kidney function, but this regimen needs to be balanced with its adverse side effects. We report in our case the improvement in kidney function with glucocorticoids and immuno-suppressive therapy which corroborate our diagnosis.

Mycophenolate and azathioprine are acceptable alternatives that have been shown to be effective in case series [15]. Biologic agents such as infliximab have been used in relapsing or steroiddependent cases [16].

\section{CONCLUSION}

The presence of AKI with kidney biopsy findings of GIN generates a broad differential diagnosis, among which renal sarcoidosis is a diagnosis of careful exclusion. Our case report recognizes granulomatous inflammation as one of the rare renal manifestations of sarcoidosis and of AKI.

\section{REFERENCES}

1. Dominique Valeyre, Antje Prasse, Hilario Nunes and all. Sarcoidosis. Lancet. 2014; 383:1155-67.

2. Newman Lee, Rose Cécile, Maier Lisa. Sarcoidosis. N Engl J Med. 1997; 336(17):12241234.

3. Bergner Raoul, Hoffmann Martin, Uppenkamp Michael. Frequency of kidney disease in chronic sarcoidosis.Sarcoidosis vasc diffuse lung dis. 2003;20(2):126-132.

4. Joint Statement of the American Thoracic Society (ATS), the European Respiratory Society (ERS) and the World Association of Sarcoidosis and Other Granulomatous Disorders (WASOG) adopted by the ATS Board of Directors and by the ERS Executive Committee, February 1999. Is J Respir Crit Care Med. 1999; 160: 736-55.

5. Deubelbeiss Und Schurmann, Gemperli Armin, Schindler Christian, Baty Florent, Brutsche Martin. Prevalence of sarcoidosis in Switzerland is associated with environmental factors. Eue Respir J. 2010; 35:1088-97.

6. Rybicki Benjamin, Iannuzzi Michael, Frederick Margaret. Familial aggregation of sarcoidosis. A case-control etiologic study of sarcoidosis (ACCESS). Am J Respir Crit Care Med. 2001; 164: 2085-91.

7. Hillerdal, Nou, Osterman, Schmekel. Sarcoidosis: epidemiology and prognosis. A 15-year European study. Am Rev Respir Dis. 1984; 130: 29-32.

8. Morimoto, Azuma, Abe. Epidemiology of sarcoidosis in Japan. Eur Respir J. 2008; 31: 372 79. 
9. Baughman, Lower, du Bois. Sarcoidosis. Lancet. 2003; 361: 1111-18.

10. Müller-Quernheim J, Schurmann M, Hofmann S. Genetics of sarcoidosis. Clin Chest Med. 2008; 29: 391-414.

11. Harzallah A, Kaaroud H, Boubaker K, Barbouch S, Goucha R, Hamida FB, Abdallah TB. Acute kidney injury with granulomatous interstitial nephritis and vasculitis revealing sarcoidosis. Saudi Journal of Kidney Diseases and Transplantation. 2017 Sep 1;28(5):1157.

12. Muther Richard, David McCarron, William Bennett. Renal manifestations of sarcoidosis.Arch Intern Med. 1981; 141(5)643-645.

13. Rajakariar, Sharples, Raftery, Sheaff, Yaqoob. Sarcoid tubulo-interstitial nephritis: long-term outcome and response to corticosteroid therapy. Kidney Int. 2006;70(1):165- 169.

14. Donald Singer, David Evans. Renal impairment in sarcoidosis: granulomatous nephritis as an isolated cause (two case reports and review of the literature). Clin Nephrol. 1986;26(5):250- 256.

15. Adam Berliner, MarkHaas, Michael Choi. Sarcoidosis: the nephrologist's perspective. Am J Kidney Dis. 2006;48(5):856-870.

16. Ahmed Mubashir, Mubashir Eisha, Dossabhoy Néville. Isolated renal sarcoidosis: a rare presentation of a rare disease treated with infliximab. Clin Rheumatol. 2007;26(8):13461349. 